\title{
Pleural Mesothelioma in a young male patient
}

\author{
A. Voulgaridis1, V. Apollonatou1, D. Lykouras 1, A. Giannopoulos¹, \\ M. Iliopoulou1 1 , K. Karkoulias ${ }^{1}$, P. Kraniotis 2 , C. Prokakis 3 , \\ M. Gkermpesi4, K. Spiropoulos 1
}

\begin{abstract}
Pleural Mesothelioma in a young male patient. A. Voulgaridis, V. Apollonatou, D. Lykouras, A. Giannopoulos, M. Iliopoulou, K. Karkoulias, P. Kraniotis, C. Prokakis, M. Gkermpesi, K. Spiropoulos.

We present the case of a 33-year-old male patient suffering from lymphocytic pleural effusion, as a result of pleural mesothelioma. Mesothelioma is a malignant tumor of the pleura that is mainly caused by chronic exposure to asbestos fibers and more than $\mathbf{4 0}$ years of exposure are needed to develop the disease. Early studies on the relationship of asbestos and mesothelioma were issued in the 1960s. Fibers migrate from the parenchyma of the lung to the visceral pleura. It is widely known that asbestos is an oncogenic factor which can cause damage to DNA. A chest $x$-ray may reveal pleural effusion with or without pleural
\end{abstract}

\begin{abstract}
thickening, whereas a chest CT may also reveal pleural thickening, uniform and/or lobular. Specific tests, such as immunohistochemical staining, are used in order to help differential diagnosis. Extrapleural pneumonectomy is used as a therapeutic option which involves removal of the lung as well as both the visceral and parietal pleura, the affected part of the pericardium and diaphragm. Surgery should be followed up by radiotherapy and chemotherapy. The surgery may lead to a mean survival rate of approximately 9-21 months. The case presented underlines that in the event of pleural effusion with a lymphocyte type physicians should consider the possibility of a pleural mesothelioma during differential diagnosis, even in relatively young patients.

Monaldi Arch Chest Dis 2013; 79: 2, 96-99.
\end{abstract}

Keywords: Pleural mesothelioma, Lung cancer, Asbestos, Malignancy.

1 Department of Pulmonary Medicine, University Hospital of Patras;

2 Department of Radiology, University Hospital of Patras;

3 Department of Thoracic Surgery, University Hospital of Patras;

4 Department of Pathology, University Hospital of Patras, Greece.

Correspondence: Kostas Spiropoulos, Professor of Pulmonary Medicine, University Hospital of Patras, Rio, Patras 26500, Greece; e-mail: spircos@upatras.gr

\section{Case Report}

A 33-year-old male smoker (10 pack years) male, a farmer without past medical history was admitted to our hospital for the investigation of fever and unilateral pleural effusion. Upon physical examination, the patient presented with pain on the left hypochondrium and $\mathrm{T}=37,4^{\circ} \mathrm{C}$, but without cough, expectoration, dyspnoea nor haemoptysis for 20 days prior to his admission. He was treated for pneumonia with a parapneumonic effusion with ampicillin/sulbactam ( $3 \mathrm{~g} \times 3)$ and moxifloxacin (400 mg x 1) daily.

A chest $\mathrm{X}$ ray $(\mathrm{CxR})$ was performed showing some opacity on the left lung. The patient subsequently underwent a chest Computed Tomography (CT) which revealed a pleural effusion with a concurrent left atelectasis and a consolidation (figures 1a, 1b, 2a, 2b). Low grade fever persisted together with night sweats. We performed thoracentesis which produced a yellowish lymphocytic exudative pleural effusion (3500 cells $/ \mathrm{mm}^{3}$ Lymphocytes: $47 \%$, Neutrophils: $3 \%$, Eosinophils: $5 \%$,
Atypical cells: 13\%, Mesothelial cells: 32\%). Therefore, an intrapleural drainage tube was inserted which drained approximately $1600 \mathrm{ml}$ of the pleural fluid. Further examinations performed included Tuberculin Skin Test $(0 \mathrm{~mm})$, Adenosine Deaminase (ADA) of the pleural fluid: 17,40 $\mathrm{IU} / \mathrm{ml}$. Gram and Ziehl Neelsen staining indicated no bacterial presence.

Bronchoscopy was carried out, without revealing any endobronchial lesions. Staining and cultures during bronchial washing for common bacteria and Mycobacterium tuberculosis as well as cytological examinations resulted negative.

The patient underwent open lung surgery and biopsy, where extensive parietal pleural masses were present in the lower-half of the hemithorax which were strongly attached to the underlying parenchyma of the lung. Biopsies were obtained from these parietal pleural masses, which were indicative of malignant tumors with morphological characteristics, namely epithelioid mesothelioma (figures 3,4 ).

A Positron Emission Tomography (PET-CT) was given in order to detect the extent of the dis- 

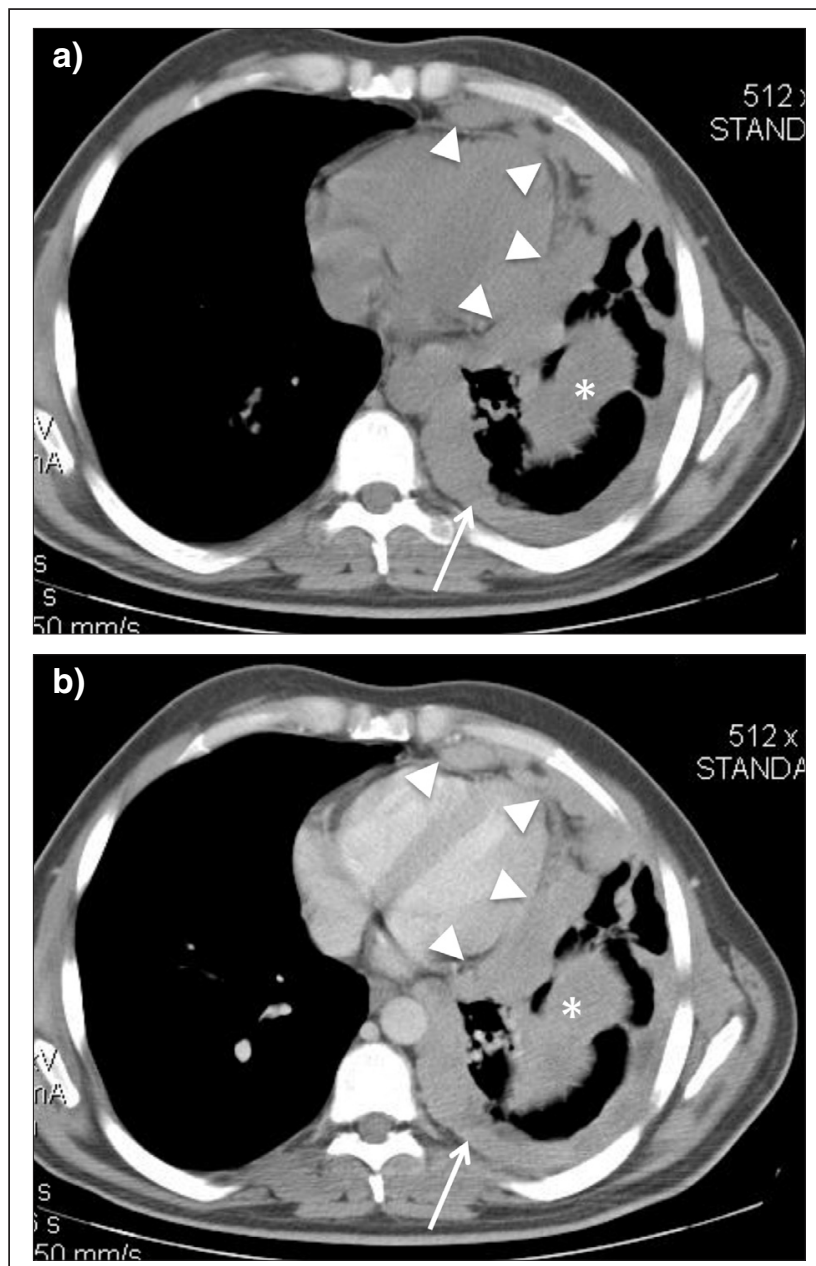

Fig. 1. - Axial CT images, with mediastinal windows at the level of the cardiac ventricles, before (a) and after (b) IV contrast. There is a marked thickening of the pleura on the left, with soft tissue density, mainly at its posterolateral/paravertebral area (white arrow), along with some rather inconspicuous tiny fluid collection along the lateral part of the pleural cavity. The thickening extends to the mediastinal part of the pleura, along the left lateral/anterolateral heart border, where lobulation and nodular lesions can be appreciated (arrowheads). The markedly thickened basal/supradiaphragmatic part of the pleura can also be seen in the middle (asterisk). The lesions are enhanced after contrast administration. There was no evidence of calcified pleural plaques, whatsoever.

ease and possible metastasis. Pathological uptake showed up on the left pleura, including both mediastinal and diaphragmatic pleura which matched the sites indicated by the chest CT scan. These lesions were in close proximity with the pericardium and the descending aorta and had invaded the leftside of the diaphragm. Moreover, the lesions had also invaded nearby tissues of the left dorsolateral peritoneum.

The patient started a course of chemotherapy with cisplatin and pemetrexed without presenting any major side effects. Specific questions about his past medical history, given the diagnosis of mesothelioma, proved that the patient had been living for more than 20 years in a warehouse which had a roof covered with asbestos.

\section{Discussion}

Mesothelioma is a malignant tumor which stems from the mesothelial (or primary sub
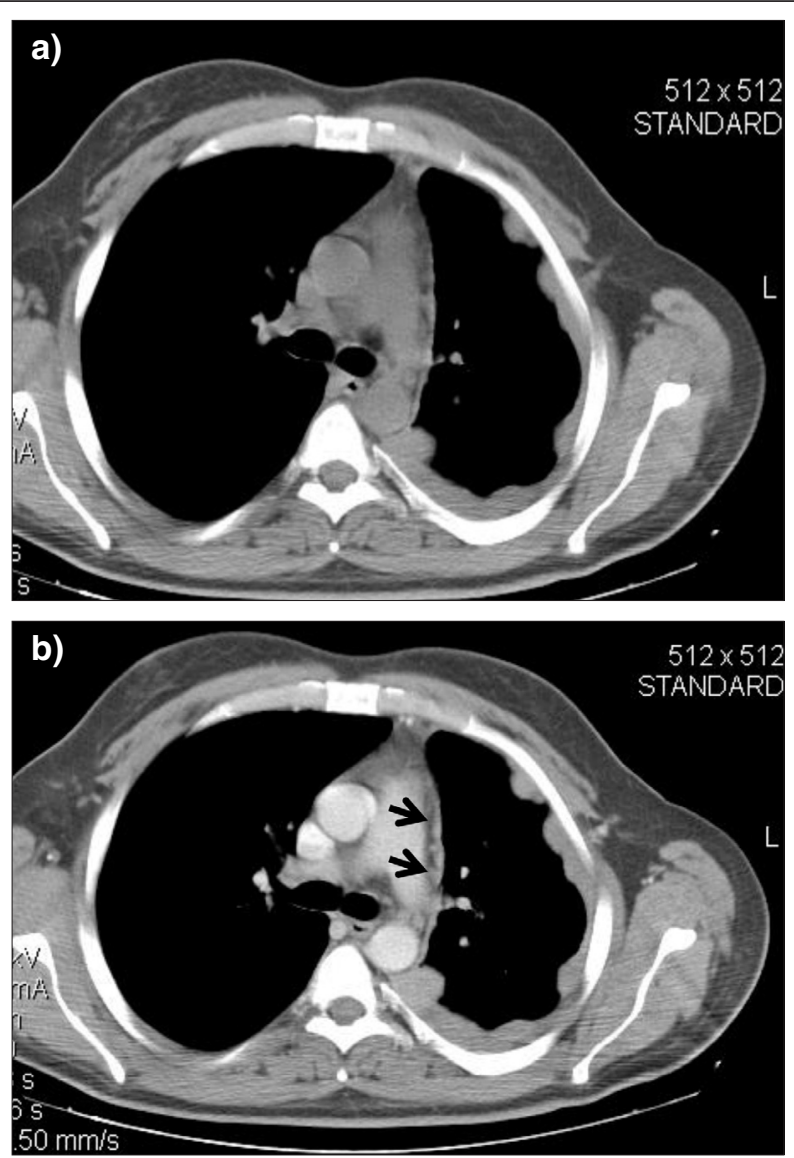

Fig. 2. - Axial CT images, with mediastinal windows at the level of the pulmonary artery trunk, before (a) and after (b) IV contrast. The pleural thickening is macronodular at the postero- and anterolateral part of the pleura. Tiny micronodular, barely discernible, lesions can be appreciated along the mediastinal pleura, along the left lateral part of the main pulmonary artery (black arrows).

mesothelial) cells. The most common anatomical site for the development of mesothelioma is the pleura $(\sim 90 \%)$, the peritoneum $(5-10 \%)$, less frequently, the pericardium $(0,4 \%)$ and the tunica vaginalis (surrounding the testis) and is caused by the exposure to asbestos.

There are many types of asbestos including chrysotile, amosite, crocidolite, anthophyllite and tremolite and exposure can be either occupational or environmental such as mining or as domestic e.g. the residents of Metsovo (Greece) who were exposed to tremolite inhalation, a material used for the whitening of walls. Early studies on the relationship between asbestos and mesothelioma were issued in the 1960s. The annual incidence of mesothelioma in people who have been exposed to asbestos is approximately 1-2 per million worldwide. It is also expected to cause more than 1000 deaths worldwide every year. The EU Directives 76/769/EEC of 2005 prohibit the extraction, processing and disposal of asbestos. The risk of developing mesothelioma is increased by prolonged exposure to asbestos, the time that has passed since first exposure to asbestos and also by the kind of the fibers involved. The average latency period between the first exposure to asbestos and death from mesothelioma is approximately 40 years; it is highly unusual to be less than 20 years and even 


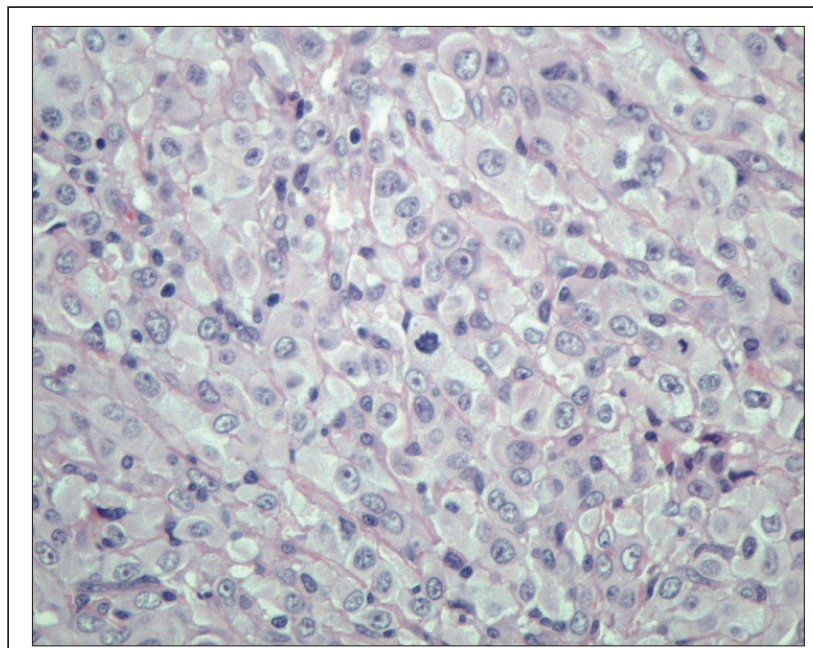

Fig. 3. - Epitheloid cells with vigorous atypia and presence of mitoses (HEX 200).

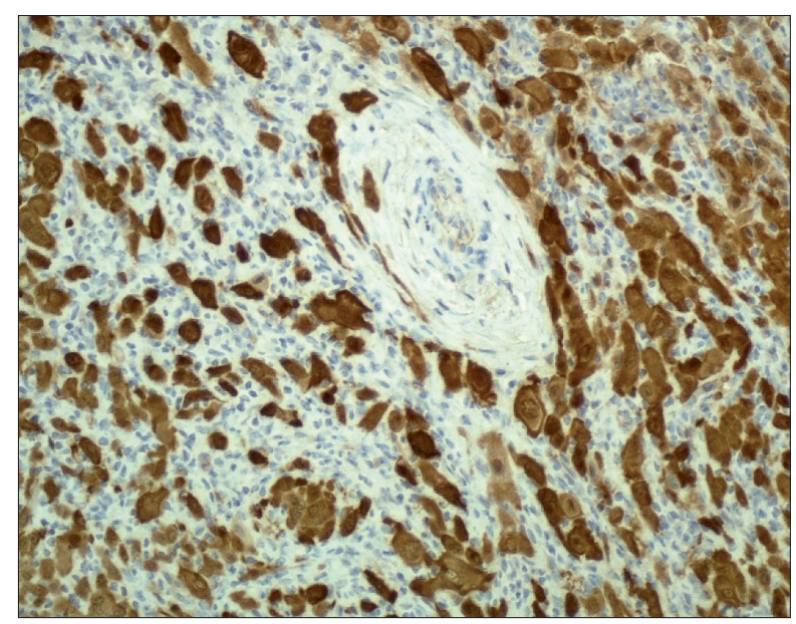

Fig. 4. - Vigorous nuclear and cytoplasmic immunostaining in immunohistochemical marker Calretinin (X200).

more unlikely to be less than 15 years. Carcinogenesis caused by asbestos exposure has been associated with the fibers that remain trapped in the lung tissue as well as their shape [1].

Fibers migrate from the parenchyma of the lung to the visceral pleura. The process of the malignant transformation has not yet been identified. However, it is well known that asbestos is an oncogenic factor which can cause DNA damage [2].

The principal types of mesothelioma are: a) epithelial $(60 \%)$, b) sarcomatoid $(10 \%)$, c) composite or biphasic (30\%). Specific tests such as immunohistochemical staining are carried out/used in order to help with differential diagnosis. Neoplastic cells are positive for CAM5.2, AE1/AE3, CK5/6, CK7, Carletinin, WT-1 thrombomodulin, HBME1, p53, EMA and negative for CEA, BerEP4 and CD15 markers.

Mesothelioma is more common in men aged between 50 and 70 years, due to occupational exposure [3]. It usually presents with rapid onset of dyspnoea due to the development of pleural effusion $(70 \%)$ and prolonged periods of chest pain $(60 \%)$. Other symptoms include anorexia, weight loss, malaise, fever and night sweats.

Unilateral pleural effusion is usually present $(95 \%)$ on the right hemithorax $(60 \%)$. Pleural pain may progressively increase due to the invasion of the malignancy. Clubbing is a rare finding (10\%), unless the patient suffers from asbestosis. Mesothelioma may spread widely attacking every organ, including contralateral pleura, the lung, intrathoracic and extrathoracic lymph nodes, the peritoneum, the liver and the brain.

A CxR may reveal pleural effusion with or without pleural thickening. Meanwhile other findings relative to asbestos exposure, such as pleural plaques, pleural thickening and asbestosis usually in the right hemithorax could well exist. A chest CT might also reveal pleural effusion (75\%), pleural thickening, uniform and/or lobular [4]. An MRI scan may help in planning the extent of infestation of the diaphragm, the mediastinum and the chest wall. PETCT can identify and classify the mesothelioma stages and help in the differentiation of benign thickening which usually shows a low intake of 2-fluoro-deoxy-D-glucose (FDG) [5]. PET is essential in indicating areas of biopsy and assessing the extent of preoperative diseases.

Extrapleural pneumonectomy is used as a therapeutic option which involves removal of the lung including both visceral and parietal pleura, the affected part of the pericardium and diaphragm. Surgery should be followed up by radiotherapy and chemotherapy.

Surgery may lead to a mean survival rate of approximately 9-21 months. Usually extrapleural pneumonectomy has a mean survival rate of approximately 13 months, while chemotherapy with radiation therapy has a mean survival rate of approximately $8-18$ months $[6,7]$. Radical surgery in the form of extrapleural pneumonectomy within trimodal therapy offers no beneficial effects and could possibly harm patients [8].

Regarding the case presented, the prevalence of mesothelioma is relatively low especially among young people. It is interesting to note that our patient was only 33 years old, which is quite uncommon for pleural mesothelioma, although it was found that he had been exposed for most of his life to asbestos in the domestic environment. The prognosis of mesothelioma, unfortunately, remains poor despite the availability of diagnostic and therapeutic options. Finally, the clinician should pay particular attention, especially in the event of pleural effusion with a lymphocyte type and should not forget the possibility of a pleural mesothelioma during differential diagnosis, even in relatively young patients.

\section{References}

1. Pelucchi C, Malvezzi M, La Vecchia C, et al. The Mesothelioma epidemic in Western Europe: an update. Br J Cancer 2004; 90: 1022-1024.

2. Kamp DW, Weitzman SA. The molecular basis of asbestos induced lung injury. Thorax 1999; 54: 638-52.

3. Hillerdal G. Malignant mesothelioma 1982; Review of 4710 published cases. Br J Dis Chest 1983; 77: 321-43.

4. Patz Jr, EF, Shaffer K, Piwnica - Worms DR, et al. Malignant pleural mesothelioma; value of $\mathrm{CT}$ and MR 
imaging in predicting respectability. Am J Roentgenol 1992; 159: 961-6.

5. Benard F, Sterman D, Smith RJ, Alavi A. Metabolic imaging of malignant pleural mesothelioma with fluorodeoxyglucose positron emission tomography. Chest 1998; 114: 713-22.

6. Nowak AK. CT, RECIST, and malignant pleural mesothelioma. Lung Cancer 2005; 49: Suppl. 1, S37S40.

7. Krug LMK, Pass H, Rusch VW, et al. A multicenter U.S. trial of neoadjuvant pemetrexed plus cisplatin (PC) followed by extrapleural pneumonectomy (EPP) and hemithoracic radiation (RT) for stage I-III malignant pleural mesothelioma (MPM). Proc Am Soc Clin Oncol 2007; 25: Suppl 20, 18S.

8. Treasure T, Lang-Lazdunski L, Waller D, et al and, MARS trialists. Extra-pleural pneumonectomy versus no extra-pleural pneumonectomy for patients with malignant pleural mesothelioma: clinical outcomes of the Mesothelioma and Radical Surgery (MARS) randomised feasibility study. Lancet Oncol 2011 12: 763-72.

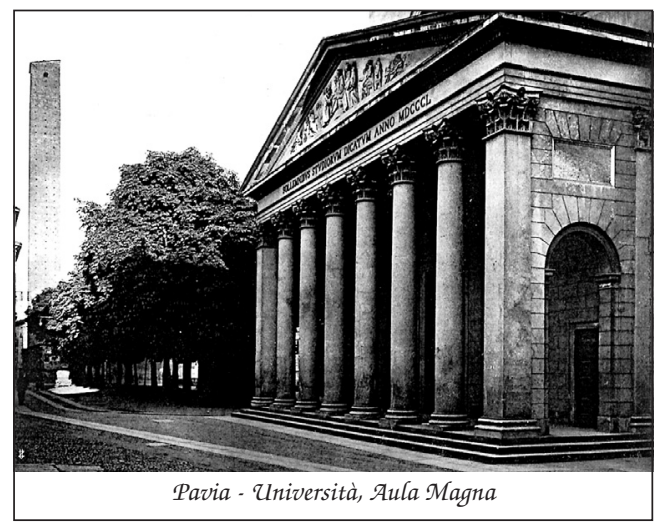

